\title{
The Telescopio San Pedro Mártir project
}

Michael G. Richer ${ }^{\mathrm{a}}$, William H. Lee ${ }^{\mathrm{b}}$, Jesús González ${ }^{\mathrm{c}}$, Buell T. Jannuzid ${ }^{\mathrm{d}}$, Beatriz Sánchez ${ }^{\mathrm{e}}$, Fabián Rosales Ortega ${ }^{\mathrm{f}}$, Charles Alcock ${ }^{\mathrm{g}}$, Alberto Carramiñana Alonso ${ }^{\mathrm{h}}$, Ma. Teresa García Díaz ${ }^{\mathrm{i}}$, Leonel Gutiérrez ${ }^{\mathrm{j}}$, Joel Herrera ${ }^{\mathrm{k}}$, Derek Hilll, Timothy J. Norton ${ }^{\mathrm{m}}$, María H. Pedrayes ${ }^{\mathrm{n}}$, Ana Pérez-Calpena ${ }^{\circ}$, Mauricio Reyes-Ruiz ${ }^{\mathrm{p}}$, Hazael Serrano Guerrero ${ }^{\mathrm{q}}$, Gerardo Sierra ${ }^{\mathrm{r}}$, Jose Teran $^{\mathrm{s}}$, David Urdaibay ${ }^{\mathrm{t}}$, Jorge A. Uribe ${ }^{\mathrm{u}}$, Alan M. Watson ${ }^{\mathrm{v}}$, Dennis Zaritsky ${ }^{\mathrm{w}}$, and Marisa García Vargas ${ }^{\mathrm{x}}$

a,i,j,k,n,p,q,r, Instituto de Astronomía, Universidad Nacional Autónoma de México, km 107 Carr. Ensenada-Tijuana, Ensenada, BC, Mexico

b,c,e,t,v Instituto de Astronomía, UNAM, Ciudad Universitaria, Mexico City, Mexico

${ }^{\mathrm{d}, \mathrm{w}}$ Dept. of Astronomy, University of Arizona, 933 North Cherry Ave., Rm. N204, Tucson, AZ, USA

f,h Instituto Nacional de Astrofísica, Óptica y Electrónica, Luis Enrique Erro \# 1, Tonantzintla, Puebla, Mexico

g,m Harvard-Smithsonian Center for Astrophysics, 60 Garden St., Cambridge, MA, USA

1,s M3 Engineering \& Technology Corp., 2051 W. Sunset Rd., Suite 101, Tucson, AZ, USA ${ }^{o, x}$ FRACTAL S.L.N.E., C/ Tulipán 2, P13-1A. E-28231 Las Rozas de Madrid, Madrid, Spain ${ }^{u}$ Centro de Ingeniería y Desarrollo Industrial, Av. Playa Pie de la Cuesta No. 702, Desarrollo San Pablo, Querétaro, Querétaro, Mexico

\begin{abstract}
The Telescopio San Pedro Mártir project intends to construct a $6.5 \mathrm{~m}$ telescope to be installed at the Observatorio Astronómico Nacional in the Sierra San Pedro Mártir in northern Baja California, Mexico. The project is an association of Mexican institutions, lead by the Instituto Nacional de Astrofísica, Óptica y Electrónica and the Instituto de Astronomía at the Universidad Nacional Autónoma de México, in partnership with the Smithsonian Astrophysical Observatory and the University of Arizona's Department of Astronomy and Steward Observatory. The project is currently in the planning and design stage. Once completed, the partners plan to operate the MMT and TSPM as a binational astrophysical observatory.
\end{abstract}

Keywords: TSPM, OAN-SPM, telescope project, optics, enclosure, site, Mexico

\section{INTRODUCTION}

The Telescopio San Pedro Mártir (TSPM) project intends to construct a $6.5 \mathrm{~m}$ telescope to be installed at the Observatorio Astronómico Nacional in the Sierra San Pedro Mártir (OAN-SPM) in northern Baja California, Mexico. The project is an association of Mexican institutions, lead by the Instituto Nacional de Astrofísica, Óptica y Electrónica (INAOE) and the Instituto de Astronomía at the Universidad Nacional Autónoma de México (IA-UNAM), in partnership with the Harvard-Smithsonian Center for Astrophysics (SAO) and the University of Arizona's Department of Astronomy and Steward Observatory (UA). The project is currently in the planning stage, with the goal of holding a preliminary design review of the enclosure in October 2016.

The telescope's mechanical design is inspired by the Magellan telescopes in Las Campanas, Chile. However, the primary mirror cell will be completely compatible with the Multiple Mirror Telescope's (MMT) Cassegrain focus. The TSPM will also include Nasmyth focal stations slightly farther from the primary than at the Magellan

Further author information: (Send correspondence to M.G.R.)

M.G.R.: E-mail: richer@astro.unam.mx, Telephone: +52 6461744580 x234

W.H.L.: E-mail: wlee@astro.unam.mx, Telephone: +52 55 5622-4020

Ground-based and Airborne Telescopes VI, edited by Helen J. Hall, Roberto Gilmozzi, Heather K. Marshall, Proc. of SPIE Vol. 9906, 99065S - (C) 2016 SPIE · CCC code: 0277-786X/16/\$18 · doi: 10.1117/12.2232000

Proc. of SPIE Vol. 9906 99065S-1 
telescopes to allow a wider field of view. This design is being lead by the Centro de Ingeniería y Desarrollo Industrial (CIDESI) in Querétaro, Mexico. Initially, the telescope is to operate in a f/5 Cassegrain configuration. The specific configurations for the future expansion to other focal stations, including Nasmyth and folded Casssegrain stations, are currently under study.

The enclosure is being designed by M3 Engineering and Technology Corp., through their branch in Hermosillo, Mexico. The design borrows elements from the Magellan telescopes (Las Campanas, Chile), but incorporating the needs of the particular site that will be used at the OAN-SPM. The primary mirror will be polished at the Steward Observatory Mirror Lab (Richard F. Caris Mirror Lab). The telescope will initially use the f/5 Cassegrain secondary currently at the Magellan II Clay telescope. The first light instruments are likely to be the Megacam and MMIRS instruments, currently at the Magellan II and MMT telescopes, respectively.

Once completed, it is planned to operate the TSPM in collaboration with the UA and SAO. Jointly, the MMT and TSPM will form a binational astrophysical observatory serving astronomers from all partner institutions, with each site focussing upon its strengths. The TSPM, thanks to its wide field imaging capability, will allow state-of-the-art research on planetary, Galactic and extragalactic astronomy, variable sky studies, and large scale surveys. TSPM will be complementary not only to the MMT for large scale, high-impact programs, but also to multi-frequency collaborations that could exploit current Mexican facilities, such as the Gran Telescopio Milimétrico Alfonso Serrano (GTM) and the High-Altitude Water Cherenkov Gamma-Ray Observatory (HAWC). The TSPM will also represent an excellent follow-up complement for the extremely large telescopes that will begin operation after 2020 .

\section{SITE}

The TSPM project will be built at the OAN-SPM, which is about $140 \mathrm{~km}$ to the southeast of Ensenada, Baja California, Mexico or about $230 \mathrm{~km}$ southeast from the Mexico-US border at San Diego, California (See Figure 1). The OAN-SPM is within the Sierra de San Pedro Mártir National Park. Currently just over 3000 hectares are zoned for observatory activities within the park. The OAN-SPM headquarters are located at the Ensenada branch of the IA-UNAM. There is daily service to the observatory on weekdays and when necessary on weekends. Given the distance from Ensenada, neither staff nor visitors commute daily. Instead, everyone resides at the OAN-SPM for the duration of their work period.
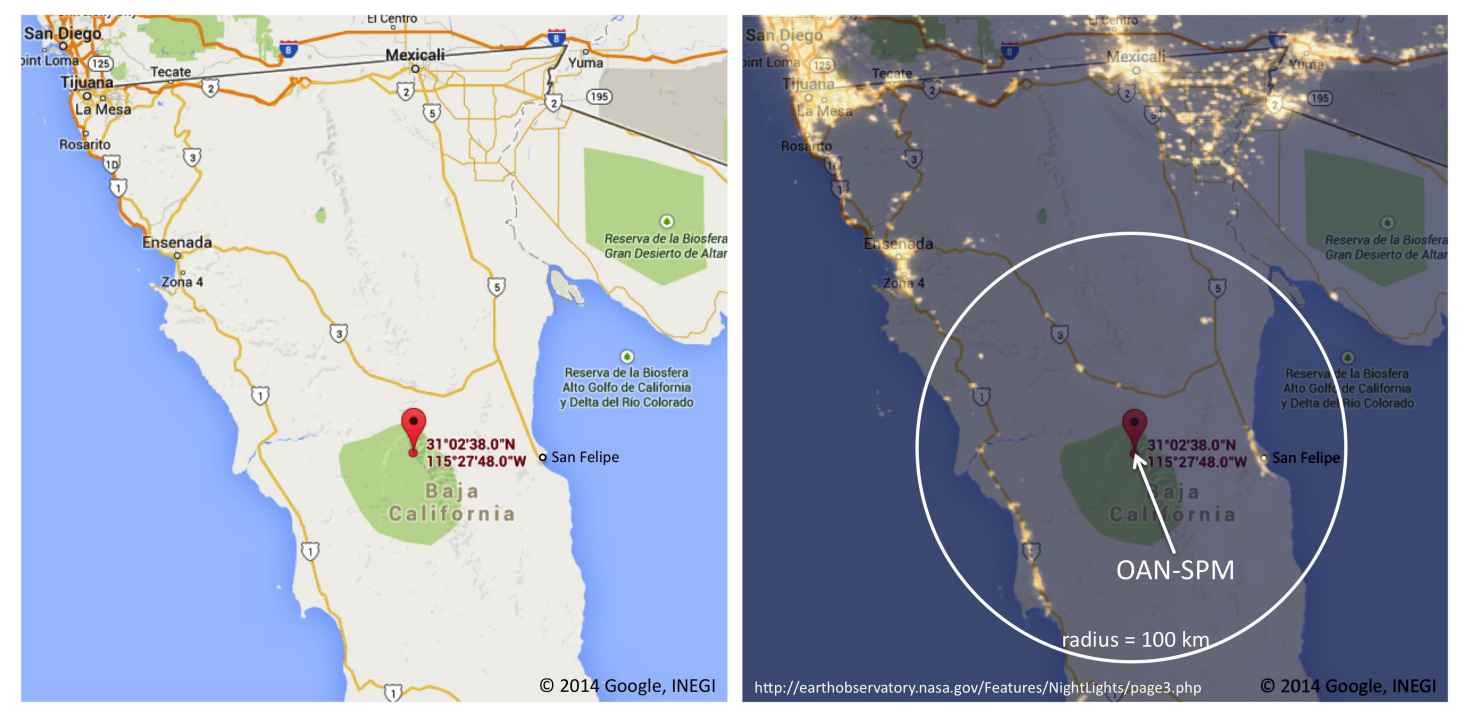

Figure 1. left panel: This map shows the north end of the Baja California peninsula and the southernmost part of California. The OAN-SPM is located approximately $140 \mathrm{~km}$ to the southeast of Ensenada, Baja California, Mexico. For reference, Ensenada is about $120 \mathrm{~km}$ south of San Diego, California. right panel: The city lights map from NASA's Earth Observatory is overlaid on the map from the left panel, illustrating the isolation of the OAN-SPM from large urban areas. There are no cities of substantial size to the south of the OAN-SPM. 
Currently, there are four operating telescopes at the OAN-SPM. These are the $84-\mathrm{cm}, 1.5-\mathrm{m}$, and 2.1-m telescopes of the OAN-SPM proper and the Böotes-5 station of the Böotes network. ${ }^{1}$ Construction is ongoing for the three $1.3-\mathrm{m}$ telescopes of the TAOS-II project. ${ }^{2}$ In addition to the telescopes, the OAN-SPM infrastructure includes a machining shop, an electronics laboratory, four diesel electricity generators, a vehicle maintenance shop, several warehouses, and the observatory lodgings that includes the dining hall, laundry and recreation facilities as well as the lodging for the day and night crews. ${ }^{3}$

Access to the OAN-SPM is via the transpeninsular highway $140 \mathrm{~km}$ south from Ensenada, then $100 \mathrm{~km}$ eastward via a paved access road that also serves ranches in the area and serves as access to the National Park. The nearest commercial ports are in Ensenada and Long Beach, California. The nearest commercial airports are in Tijuana and San Diego, California (Figure 1).

Currently, the OAN-SPM is not connected to the national electricity grid. All power is generated by diesel generators on-site. One of these generators is configured with transfer capability and comes online within seconds if power is lost. Telecommunications are provided by a $10 \mathrm{Mbit}$ microwave link towards the east side of the Baja California peninsula. From the sea level end of the link, signals travel via fibre optics to Ensenada. A connection to the national electricity grid is currently under construction. Once completed in 2017, this connection will also include a fibre optics connection directly to the OAN-SPM.
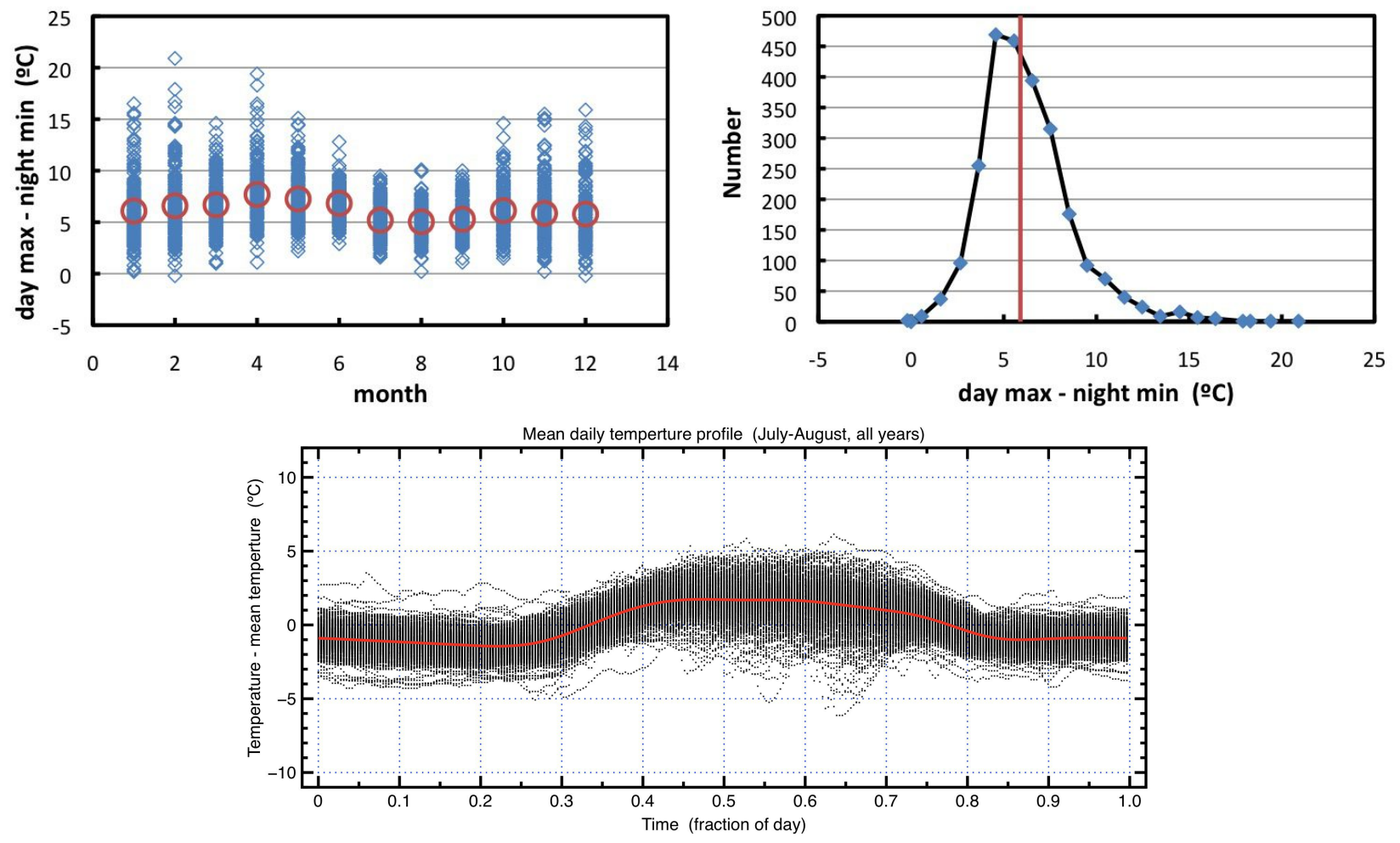

Figure 2. top left: We plot the difference in temperature between the daytime high and night-time low as a function of the month for all days from June 2006 to August 2013. The circles represent the mean values for each month of the year. There is clearly more variation in the daily temperature excursion in winter than in summer. top right: We present the histogram of all values from the left panel. Overall, the median of the daily temperature excursion is $5.9^{\circ} \mathrm{C}$. bottom: We present the mean temperature profile during the day for the months of July and August (all temperature measurements) for the same period. This plot clearly demonstrates that there is very little temperature variation from an hour after sunset until sunrise.

There is considerable information on the climate and observing conditions at the OAN-SPM by both local efforts $^{4-11}$ and the Thirty Meter Telescope group. ${ }^{12-15}$ Generally, studies find of order $70 \%$ of the nights are photometric and $80 \%$ are clear (spectroscopic), ${ }^{9,11}$ with summer having less clouds than winter. The atmospheric 
extinction is low and stable. ${ }^{6}$ The median seeing at $5000 \AA$ is $0.79^{\prime \prime}$ and dominated by the lowest atmospheric layer. ${ }^{8,13}$ The median precipitable water vapour is $3.4 \mathrm{~mm}$, with the site being substantially drier in winter. ${ }^{15}$ The winds are not too strong and show only a modest preference of coming from either the south or south-southwest and north or north-northeast. ${ }^{5,10}$ Wind from either the east or west is less common. Analyses undertaken by the project indicate that the median temperature swing from daytime high to night-time low is $5.9^{\circ} \mathrm{C}$, that this daily temperature excursion is smallest in summer, and that the nights have small temperature gradients from one hour after sunset to sunrise (see Figure 2). Typically, the temperature is lowest at sunrise and it correlates well with the temperature at sunset later the same day, at least for sunrise temperatures higher than $0^{\circ} \mathrm{C}$ (see Figure 3).

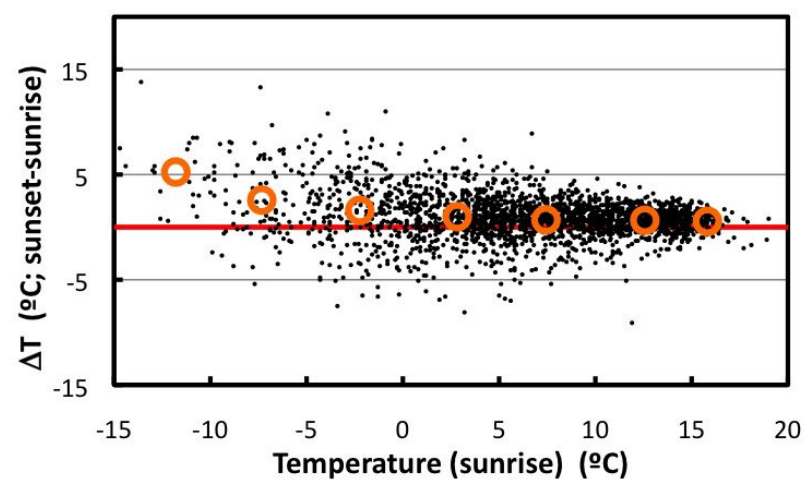

Figure 3. We present the difference in temperature between sunset and sunrise on a given day as a function of the temperature at sunrise. The circles indicate the mean value in each $5^{\circ} \mathrm{C}$ bin. For sunrise temperatures exceeding $0^{\circ} \mathrm{C}$, the mean difference is less than $1^{\circ} \mathrm{C}$.

The project has recently commissioned geophysical and geological studies of the proposed construction site. The study was done by the Gerencia de Estudios de Ingeniería Civil of the Comisión Federal de Electricidad (national electricity company). The immediate purpose of this study is to optimize the requirements for the telescope pier, building foundations, and roadways.

\section{OPTICAL DESIGN}

The base configuration of the TSPM uses a $6.5 \mathrm{~m}$ honeycomb primary mirror (M1) cast by the University of Arizona's Richard F. Caris Mirror Lab and polished to the same prescription and specifications as the primaries of the MMT, Magellan, and Tokyo Atacama Observatory (TAO $)^{16}$ telescopes. The M1 cell will be nominally identical to that at the MMT, though in practice it is a copy of the TAO design. In particular, the design incorporates a cone-shaped opening to the Cassegrain derotator. The M1 assembly is a contribution from INAOE and the UA.

The TSPM will use the existing f/5 secondary from the Magellan II (Clay) telescope together with its widefield corrector (WFC). ${ }^{17}$ The UA and SAO are contributing these components to the project.

The "Day 1" optical configuration is thus equivalent to the $\mathrm{f} / 5$ Cassegrain configurations at the MMT and the Magellan II (Clay) telescopes. Two other optical configurations are currently under study that affect the telescope structure and the dome (see Figure 4). Since the long-term goal is that the TSPM be dedicated to wide-field uses, one of the post-Day 1 optical configurations under study is f/5 Nasmyth (Figure 4 ). This optical configuration affects the telescope structure due to the large tertiary mirror required, which forces the optical/elevation axis away from the primary mirror. The other post-Day 1 optical configuration being studied is an $\mathrm{f} / 11$ Gregorian configuration, also at Nasmyth, as used at the Magellan telescopes. This configuration has an important impact on the size of the dome (Figure 4). Table 1 provides further details of the three configurations.

The $\mathrm{f} / 5$ Cassegrain configuration is nominally an exact copy of Magellan and MMT designs. A full optical design and error budget analysis is underway for this configuration. For the TSPM, the high level requirements 
Table 1. Optical Design.

\begin{tabular}{|c|c|c|c|}
\hline & $\mathrm{f} / 5$ Cass & f/5 Cass@Nas** & f/11 Gregorian@Nas** \\
\hline Primary Mirror (M1) & \multicolumn{3}{|c|}{ Diameter $=6502 \mathrm{~mm}, \mathrm{R}_{\mathrm{c}}=16256 \mathrm{~mm}$, Conic $=-1.0$} \\
\hline Elevation axis & \multicolumn{3}{|c|}{$1,000 \mathrm{~mm}$ above $\mathrm{M} 1$ vertex } \\
\hline Eff. focal length $(\mathrm{mm})$ & 34372.5 & 34358.8 & 71524 \\
\hline FoV (degrees) & \multicolumn{2}{|c|}{ 1.0/0.5 spectroscopy/imaging } & 0.45 \\
\hline M1-M2 distance (mm) & 6184.65 & 5824.4 & 9671.6 \\
\hline M2 diameter $/ R_{\mathrm{c}}(\mathrm{mm})$ & $1682 /-5151.64$ & $1966 /-6045.7$ & $1308 / 2777.4$ \\
\hline M2 conic & -2.695 & -2.61519 & -0.63888 \\
\hline M3 location* (mm) & & \multicolumn{2}{|c|}{$(0,-46.23, \quad-46.23) \quad(65.38 \mathrm{~mm} \quad \operatorname{disp})$} \\
\hline M3 aperture & & \multicolumn{2}{|l|}{$1830 \times 1300$ ellipse } \\
\hline WFC concept & \multicolumn{2}{|c|}{3 -elements +2 -doublet ADC } & 2-doublet ADC \\
\hline Focal surface location* $(\mathrm{mm})$ & $(0,0,2713.4)$ & $(0,4885,0)$ & $(0,5100,0)$ \\
\hline Focal surface $\mathrm{D} / \mathrm{R}_{\mathrm{c}}(\mathrm{mm})$ & $611.3 /-3404$ & $611.3 /-5278$ & $611.3 / 1214$ \\
\hline
\end{tabular}

* Locations are relative to the coordinate-system center defined by the intersection of the telescope optical axis and the elevation axis (1.0 $\mathrm{m}$ above the primary vertex).

** These optical prescriptions are schematic, to define the extremes of the telescope and enclosure designs. They will be refined after definition of future science cases and instrumentation.

stipulate that the delivered image quality shall allow a $10 \%$ degradation of the 10 th-percentile seeing, ${ }^{13}$ that the telescope shall be optimized from the near ultraviolet to the near infrared $(0.35-2.5 \mu \mathrm{m})$, and that two Nasmyth and four folded Cassegrain focal stations compatible with the $\mathrm{f} / 11$ and $\mathrm{f} / 5$ configurations be available, all equipped with a mechanical de-rotator, guide sensor and WFS similar to the existing sensors in the existing $\mathrm{f} / 5$ Cassegrain. For the Nasmyth focal stations, the minimum unobscured focal surface is specified as $0.60 \mathrm{~m}$ in diameter while, for the folded Cassegrain focal stations, it is $0.30 \mathrm{~m}$ in diameter.

Table 2 presents details of the error budget for the $\mathrm{f} / 5$ Cassegrain configuration, incorporating the prescription for the primary mirror figure as well as the as-built parameters of the secondary mirror and WFC. At present, this study has yet to optimize completely all of the parameters involved, but it is clear that the principal components are all due to optics and their alignment. Figure 5 presents the computed spot diagrams for the $\mathrm{f} / 5$ Cassegrain configuration for different positions and wavelengths of interest.

\section{TELESCOPE DESIGN}

The general design of the telescope structure is inspired by the Magellan telescopes. ${ }^{18}$ The same basic tripod disc design is adopted as a starting point for the TSPM design. As in the Magellan design, all axes are driven by friction drives and hydrostatic bearings are used for the azimuth and elevation axes. The telescope design is being done by the Centro de Ingeniería y Desarrollo Industrial, a Mexican federal research institute in Querétaro, Mexico.

The main differences with respect to the Magellan design are (Day 1 configuration) the interface to the primary mirror cell for the Cassegrain focal station, the position of the elevation axis, the capacity of the Nasmyth platforms, and the interface to the secondary mirror. ${ }^{19}$ Since the project partners envisage operating the TSPM and MMT in a complementary way, the backside of the primary mirror cell will be identical to that of the MMT. This will permit the exchange of instruments for the $\mathrm{f} / 5$ Cassegrain focal stations at both telescopes. As noted above, the larger tertiary mirror needed for the $\mathrm{f} / 5$ Nasmyth focal station forces the position of the elevation axis farther from the primary mirror than at the Magellan telescopes. As a consequence, the discs and 


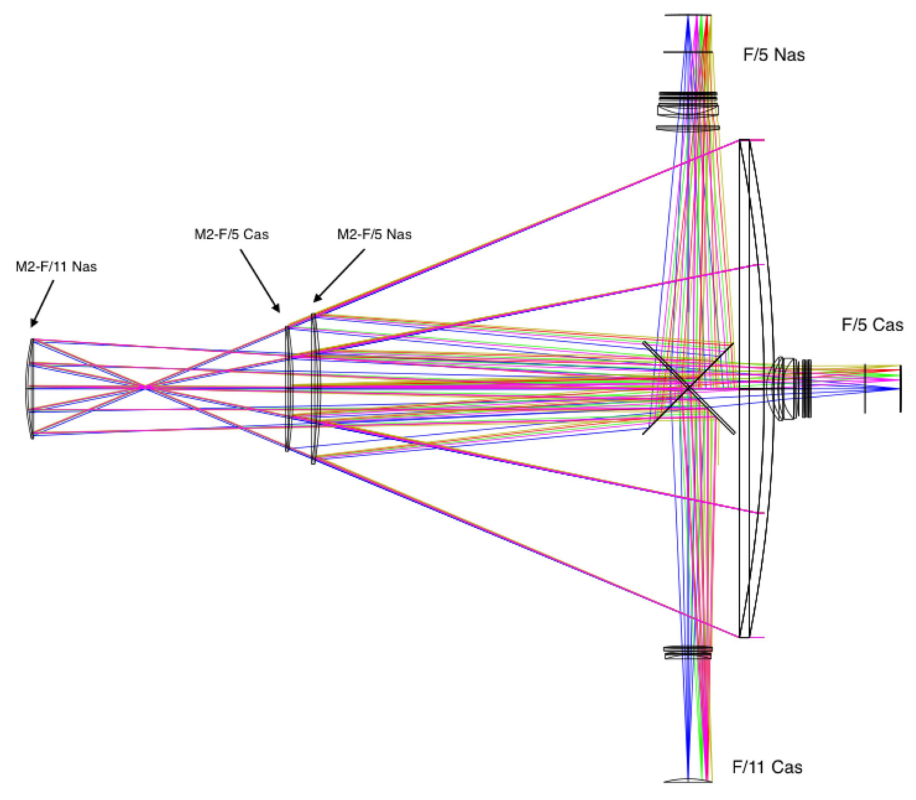

Figure 4. We present the TSPM optical configurations considered in the project design. The f/5 Cassegrain configuration will be the "Day 1" configuration. The other configurations are indicative and used to dimension the building and telescope structure. In particular, the f/5 Nasmyth configuration drives the size of M3 and the telescope structure while the f/11 Gregorian configuration drives the height of the dome.
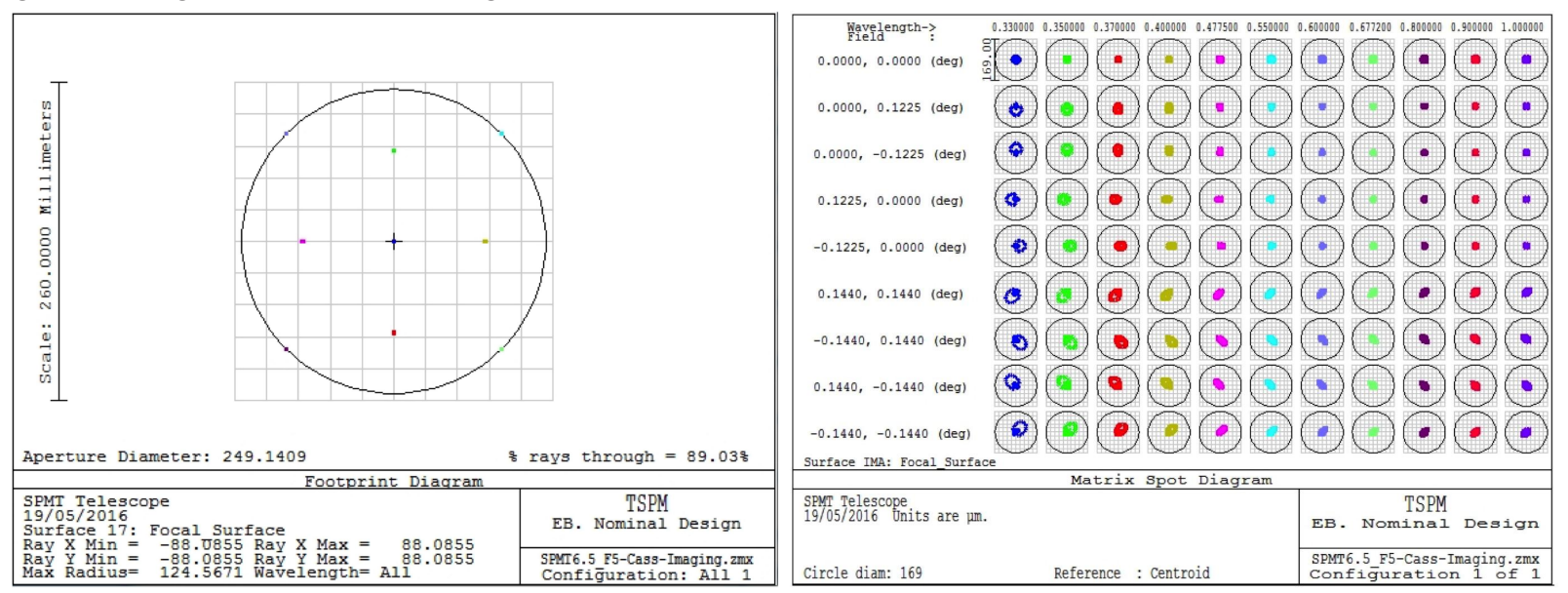

Figure 5. left: We present the positions used to compute the spot diagrams dispersed over the field corrected by the Cassegrain wide field corrector (WFC). right: The spot diagrams for 11 wavelengths over the $0.35-1.0 \mu \mathrm{m}$ interval. The circle size corresponds to an angular size of $1^{\prime \prime}$. Note that there is no atmospheric dispersion corrector for the imaging configuration.

tripods used in the TSPM design are also larger. The Nasmyth platforms are larger and have a larger capacity than those of the Magellan telescopes as a result of the high level design requirements. Finally, the telescope design currently envisages using a hexapod to control the secondary mirror, as is used at the MMT, rather than the vane-end system used at Magellan. ${ }^{18}$

\section{ENCLOSURE}

The site chosen for the TSPM project is at the western end of the ridge where the OAN-SPM's three telescopes are located (see Figure 6). This ridge is nearly perpendicular to the typical wind directions, though these are 
Table 2. f/5 Cassegrain Error Budget.

\begin{tabular}{|c|c|}
\hline Image Quality & rms (arcsec) \\
\hline 1. Seeing & 0.502 \\
\hline 1.1 Atmospheric seeing & 0.500 \\
\hline 1.2 Seeing degradation (telescope, dome, mirrors) & 0.040 \\
\hline 2. Nominal optical configuration & 0.140 \\
\hline 3. Telescope and optics errors & 0.223 \\
\hline 3.1 M1 manufacturing & 0.179 \\
\hline 3.1.1 M1 conic/radius manufacturing & 0.056 \\
\hline 3.1.2 M1 high frequency errors manufacturing & 0.170 \\
\hline 3.2 M2 manufacturing & 0.049 \\
\hline 3.2.1 M2 conic/radius as-built measurement & 0.028 \\
\hline 3.2 .2 M2 high frequency errors manufacturing & 0.040 \\
\hline 3.3 WFC manufacturing & 0.065 \\
\hline 3.4 Optical Alignment & 0.099 \\
\hline 3.4.1 M1 alignment & 0.036 \\
\hline 3.4.2 M2 alignment and focus & 0.090 \\
\hline 3.4.3 WFC alignment & 0.018 \\
\hline 3.5 Guiding & 0.030 \\
\hline 3.6 M1 thermal variations & 0.020 \\
\hline Total & $\mathbf{0 . 5 6 6}$ \\
\hline Requirement & $\mathbf{0 . 5 5 0}$ \\
\hline
\end{tabular}

not very strongly defined. ${ }^{5,7,10}$ The enclosure design has been contracted to M3, specifically its affiliate office in Hermosillo, Mexico. ${ }^{20}$ As for the telescope structure, the design of the enclosure is also inspired by the Magellan design.

A fundamental design requirement that strongly influences the enclosure design is the choice of setting the telescope's elevation axis $12 \mathrm{~m}$ above the local terrain. Studies have shown ${ }^{8}$ that the ground layer turbulence falls significantly above $10 \mathrm{~m}$ above the local ground level, a consideration that sets a minimum height for the elevation axis. The relationship between the telescope enclosure and the support building then follows based upon the intention to emulate the mirror handling followed at the Magellan telescopes, which requires that the upper level of the telescope enclosure be at the same elevation as the upper level of the support building. The current design has the upper level of the support building meet the ground level at the northeast end of the support building, thus facilitating the handling of instruments and other major components.

The telescope enclosure is of very similar size to that of the Magellan telescopes, with the clearance radius of the dome being $9.8 \mathrm{~m}^{22}$ On the upper level is the telescope chamber and the lower level will include a spectrograph room and a few minimal support facilities that must be located there. The telescope enclosure is connected to the support building via a bridge (for instruments and mirrors) and a tunnel (for people).

The lower level of the support building is devoted to the night-time use of telescope and contains the control room, computer and IT rooms, offices, and other amenities. The upper level of the support building is devoted to the daytime needs of the telescope and instruments. Access to the telescope chamber is provided by the bridge between the two buildings, over which instruments will travel when these are changed as well as the mirrors when they are aluminized. The main entrance on the front of the support building (northeast end) will allow 


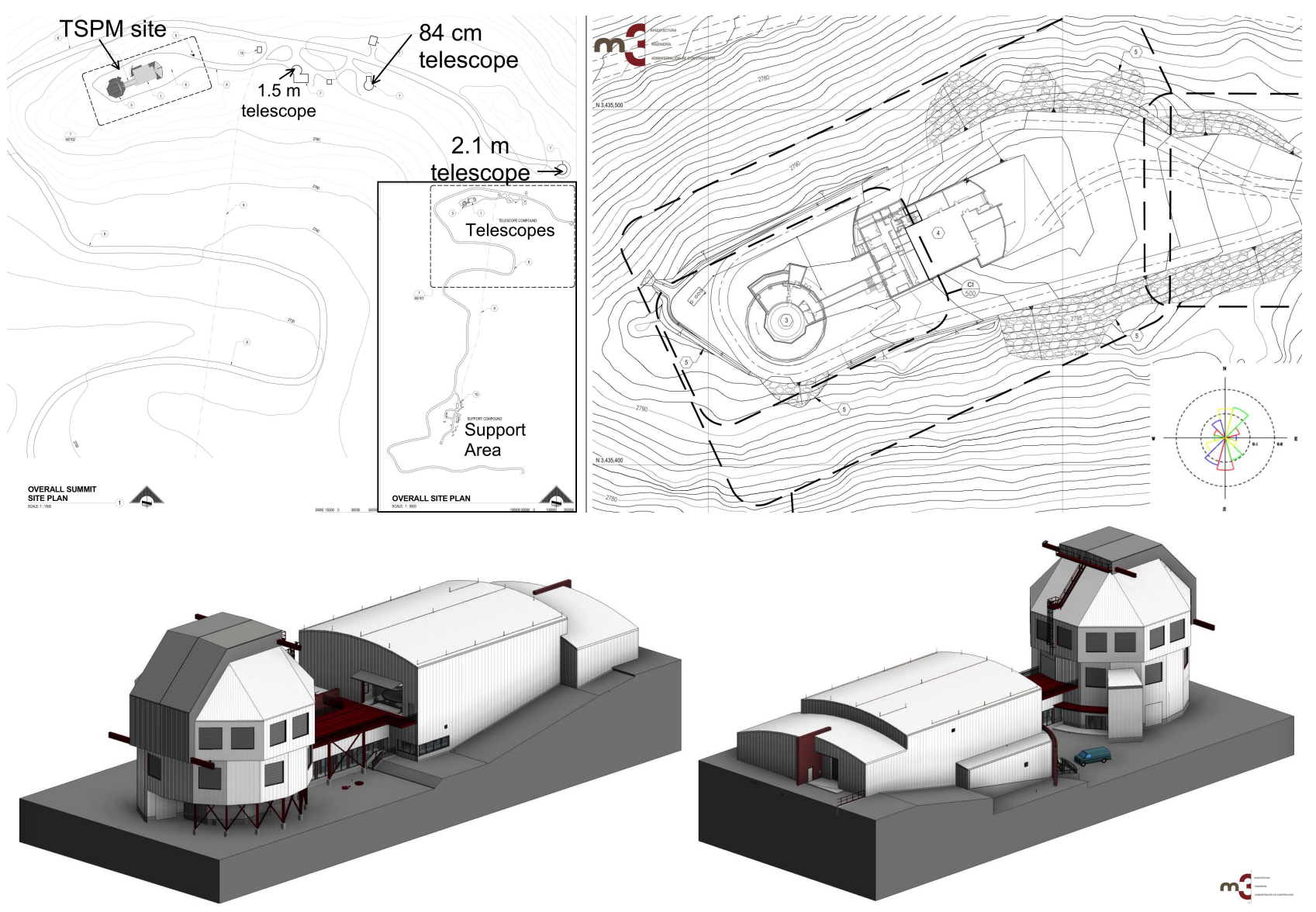

Figure 6. top left: The main map provides an overview of the telescope area at the OAN-SPM while the inset shows a larger area (but not including the area where the TAOS-II project is being built, to the east). The telescopes occupy a narrow ridge that runs roughly east-west. top right: The outline of the TSPM enclosure is overlaid upon a topographical map of the future construction site. The orientation of the telescope building and support building takes advantage of the shape of the terrain, which is highest where the support building is located. A typical wind rose (night-time) is shown at lower right, ${ }^{10}$ from which it is clear that the wind is usually at a substantial angle to the proposed orientation of the enclosure. bottom: 3-D models of the enclosure as viewed from the south (left) and north (right). The main entrance to the support building (bottom, right) is sized to accomodate envelopes as large as those that fit in an industrial container.

unloading equipment whose envelope matches that of industrial containers. There is still some discussion of where to locate the hydraulic pumping plant as well as the emergency generator, and it is possible that they may be located in a separate underground room to the west of the telescope enclosure.

\section{INSTRUMENTATION}

At present, the Day 1 instruments planned for the TSPM are the Megacam ${ }^{23}$ and then MMIRS ${ }^{24}$ instruments that currently operate at the $\mathrm{f} / 5$ Cassegrain focal stations at the Magellan and MMT, respectively. We envisage the commissioning proper to occur with Megacam and, once the most important issues have been worked out, MMIRS could then be brought online. In this way, the TSPM would begin its scientific operation with a brightand a dark-time instrument. Both instruments are a contribution of the SAO.

The TSPM science working group is fleshing out plans for both the Day 1 instruments and instruments to arrive afterwards, including new instruments to be built in Mexico. 


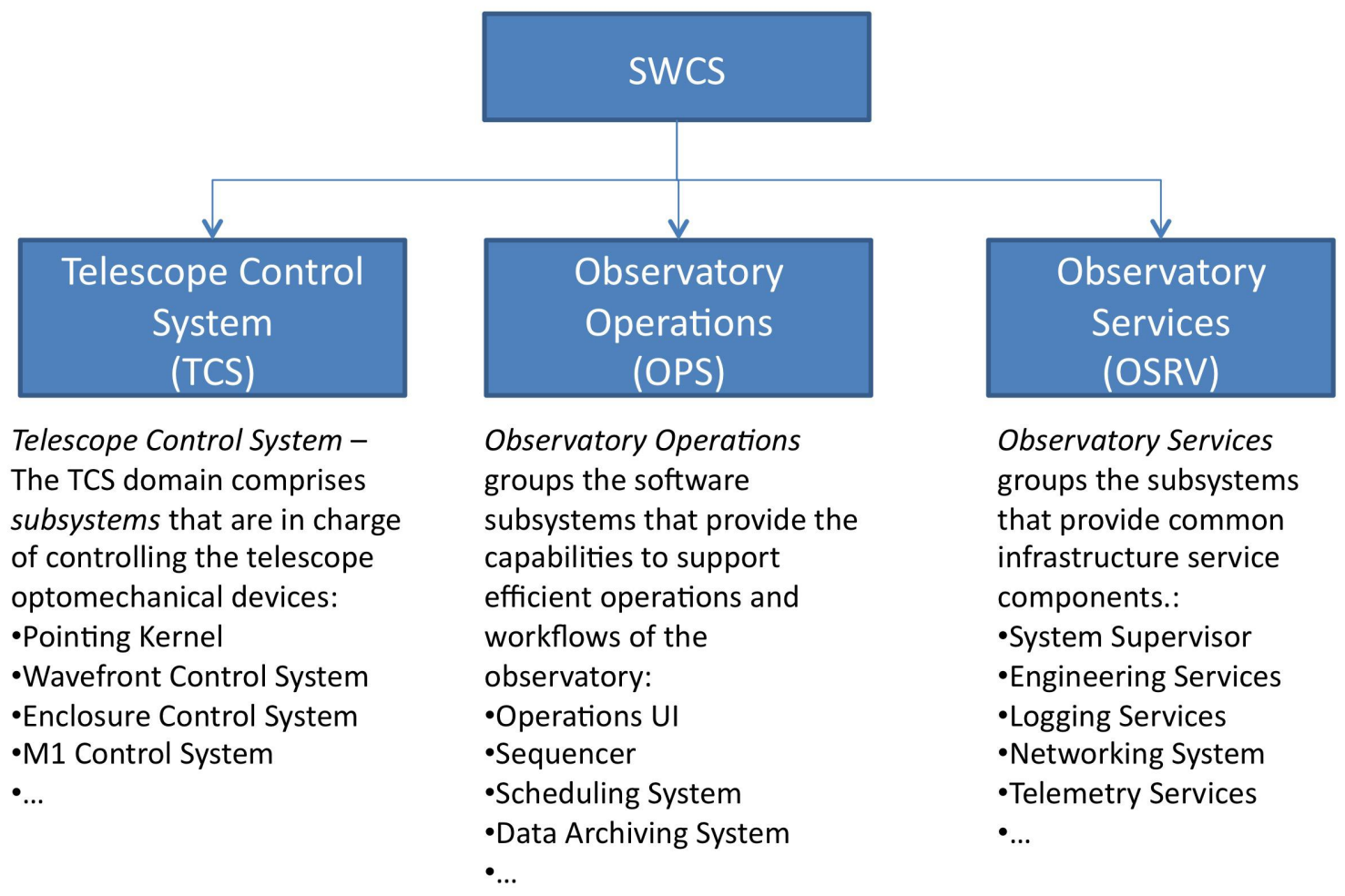

Figure 7. This diagram presents a schematic view of the proposed project software control system (SWCS), based upon that developed for the Giant Magellan Telescope. ${ }^{21}$ Its functions are broken down into three general domains.

\section{CONTROL SYSTEM}

After reviewing various examples of software control systems, the TSPM project decided that the system adopted by the Giant Magellan Telescope ${ }^{21,25}$ was the most appropriate for its needs in a general sense. Although very ambitious in its scope, this system allows incorporating the different needs of the different user communities involved in the project.

Figure 7 provides an overview of the general structure of the software and control system being contemplated. Generally, its functions are divided among three broad areas: observatory operations, observatory services, and telescope control. Observatory operations includes all activities related to realizing scientific observations and observatory maintenance. Observatory services provides a common infrastructure to accommodate the communication and storing of necessary information. Finally, telescope control takes care of all operations required to acquire scientific data or calibrations.

Not all of the components will necessarily fit precisely in this scheme. For instance, the primary mirror cell, the instruments, and other components will be provided with control systems that obey different philosophies. The TSPM project plans on accommodating this diversity by providing "interface layers" for those specific components, as is already done for Megacam and MMIRS at the MMT and Magellan.

\section{OPERATION}

The IA-UNAM, INAOE, UA and SAO have the intent to contribute different elements to the TSPM project, namely: UNAM will provide the site and associated infrastructure, INAOE and UA will provide the primary mirror system, UA and SAO will provide the M2 and corrector optics, and first light instruments (in particular, but not limited to Megacam and MMIRS), and Mexico will develop the telescope structure and enclosure and support buildings. Thus far, the design phase of TSPM has been funded primarily by Mexico's Consejo Nacional de Ciencia y Tecnologia (CONACyT). Once completed, the TSPM will serve both user communities and the 


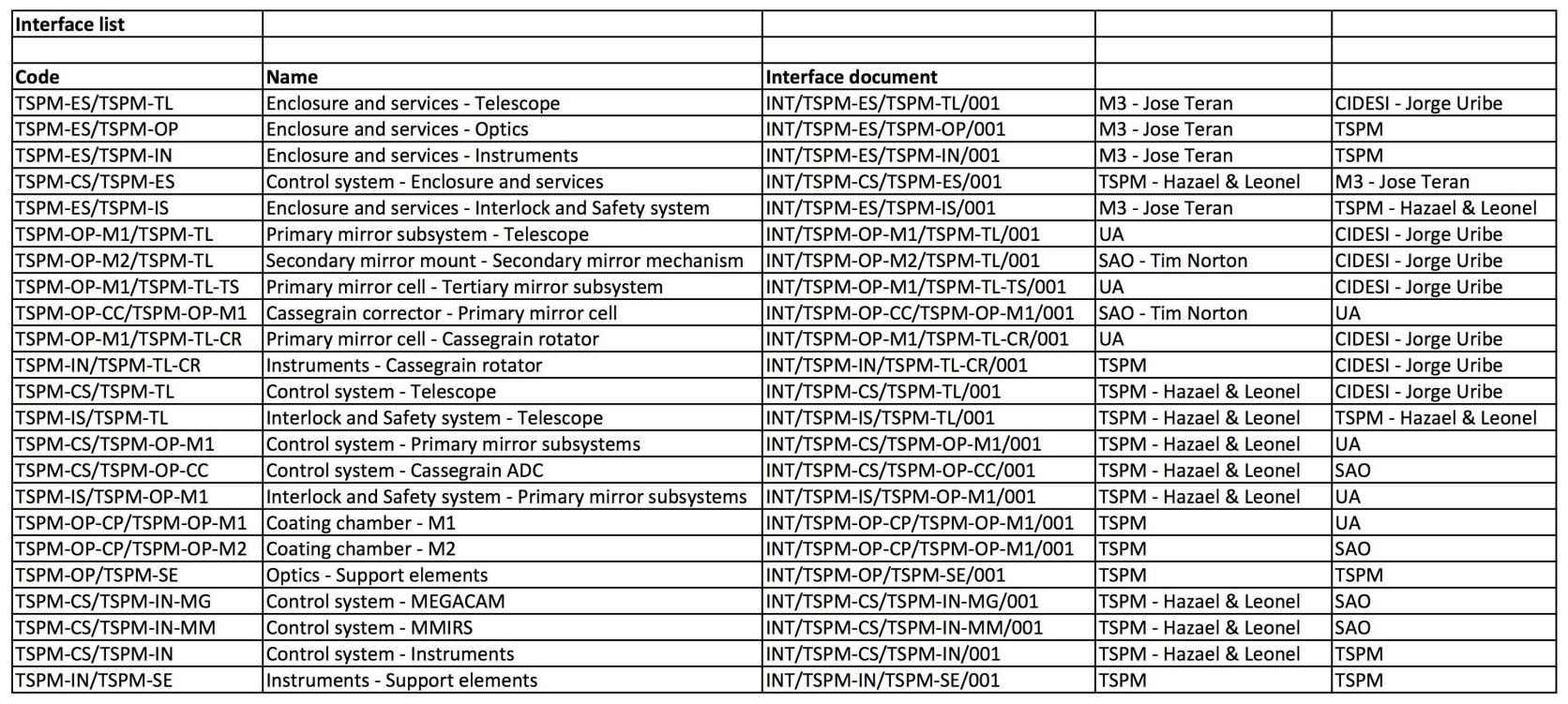

Figure 8. Above, we present a list of the interfaces currently under development.

MMT will be accessible to the wider Mexican community for use, with each national party being responsible for the operation of the telescope in its own territory. Thus, the TSPM and the MMT will operate as a bi-national Mexico-US astrophysics laboratory, with the goal of carrying out high impact research in astrophysics by taking advantage of the natural qualities and operating schemes at each of the two sites, and carry out programs that will complement those of 20-30 m class telescopes currently under design and construction.

\section{SYSTEMS ENGINEERING AND PROJECT MANAGEMENT}

The TSPM project has contracted the systems engineering and project management with FRACTAL S.L.N.E.

At this point, the project's high level requirements have been fixed in order to explore whether any issues are insurmountable at a reasonable cost while maintaining high performance. A system-level specification has been developed and subsystem-level specifications are being derived from it. Interfaces are also being generated between subsystems that will provide the boundaries to be considered by the different groups involved in each interface. For a list of the current interfaces, see Figure 8. We are currently working on different error and technical budgets.

Figure 9 presents a schematic calendar for the project. The dates are undoubtedly speculative, but this calendar's main purpose is to plan the steps required to complete the project.

The current estimated date for Day 1 is 26 December 2022. Day 1 implies that the $\mathrm{f} / 5$ configuration is fully commissioned and Megacam is in operation. MMIRS would come six months after that. The project calendar is continuously updated in the MANATEE tool and is based on individual milestones that are linked to their parent ones. (We are using the suite of project management tools provided by FRACTAL S.L.N.E..) One milestone can be linked to several others and the program automatically calculates the longest path towards a given date, the so-called critical path. Table 3 shows the critical path towards Day 1. MANATEE provides information not only on the milestones belonging to the critical path but also on the milestones that are near it and could become critical. This utility will be used to assign priorities and to avoid deviations from the calendar.

The project calendar will depend sensitively on the funds and the associated cash-flow. In fact, the project will be driven by the budget and the cash-flow, and at this moment cannot be considered a schedule-driven project.

The budget is composed of the monetary and the in-kind contributions. The latter are very important in this project, in terms of real hardware, such as the $\mathrm{f} / 5$ Cassegrain secondary mirror, the $\mathrm{f} / 5$ Cassegrain WFC, the 


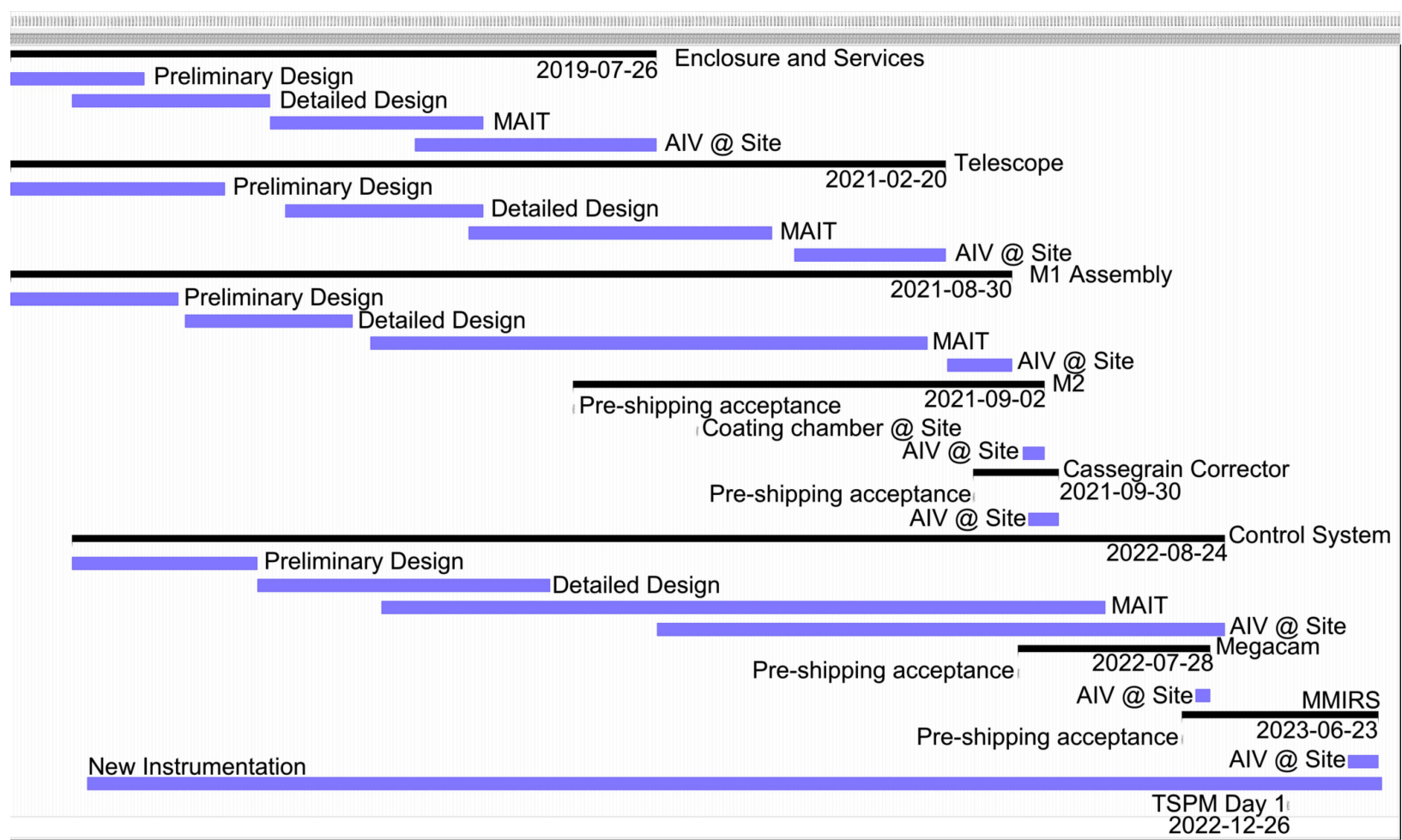

Figure 9. This figure presents a schematic version of the proposed project calendar. While the dates are speculative, its purpose is to establish all of the steps required to complete the project.

instruments from SAO, the M1 assembly from UA/INAOE, and the human resources at the different partner institutions. The monetary contribution for Day 1 is estimated at US $\$ 70 \mathrm{M}$, which will be requested from CONACyT on a year-by-year basis.

\section{ACKNOWLEDGMENTS}

The funding for this planning phase of the TSPM project has been supported in Mexico by the IA-UNAM, the INAOE, and especially the Consejo Nacional de Ciencia y Tecnología through grants LN127736, LN232649, LN 260369, LN 271117, 224003, 264173, and the Agencia Espacial Mexicana who contributed to grant 248500. We gratefully acknowledge the Giant Magellan Telescope for permitting the TSPM project to adopt its control system as our model. We thank personnel at the MMT and Magellan telescopes for making information available.

\section{REFERENCES}

[1] Castro-Tirado, J. A., Soldan, J., Bernas, M., Páta, P., Rezek, T., and et al., "The Burst Observer and Optical Transient Exploring System (BOOTES)," Astronomy 83 Astrophysics Supplements 138, 583-585 (1999).

[2] Lehner, M. J., Wang, S.-Y., Alcock, C. A., Cook, K. H., Fureszc, G., and et al., "Status of the Transneptunian Automated Occultation Survey (TAOS II)," in [Ground-based and Airborne Telescopes V], Stepp, L. M., Gilmozzi, R., and Hall, H. J., eds., Proc. SPIE 9145, 914513 (2014).

[3] López, J. A. and Gutiérrez, L., "A Primer for the San Pedro Mártir Observatory," in [San Pedro Mártir: Astronomical Site Evaluation], Cruz-Gonzlez, I., Avila, R., and Tapia, M., eds., Revista Mexicana de Astronomía y Astrofísica (Serie de Conferencias) 19, 3-7 (2003).

[4] Alvarez, M. and Maisterrena, J., "Climatological and Meteorological Characteristics of the Observatorio Astronómico Nacional at San Pedro Mártir, B.C.," Revista Mexicana de Astronomía y Astrofísica 2, 43-52 (1977). 
Table 3. Critical Path towards Day 1.

\begin{tabular}{|l|l|l|}
\hline Date & Milestone & Length \\
\hline $2022-12-26$ & Day 1: TSPM in operation with Megacam & 1 \\
\hline $2022-12-25$ & COM: Commissioning Phase I. End & 180 \\
\hline $2022-06-28$ & COM: Commissioning Phase I. Start & 1 \\
\hline $2022-06-27$ & AIV: Integration at Site. M1-M2 Alignment. End & 60 \\
\hline $2022-04-28$ & AIV: Integration at Site. M1-M2 Alignment. Start & 1 \\
\hline $2022-04-27$ & AIV: Integration at Site. Control System-M2 subsystem. End & 90 \\
\hline $2022-01-27$ & AIV: Integration at Site. Control System-M2 subsystem. Start & 1 \\
\hline $2022-01-26$ & AIV: Integration at Site. Control System-M1 assembly. End & 180 \\
\hline $2021-07-30$ & AIV: Integration at Site. Control System-M1 assembly. Start & 1 \\
\hline $2021-07-29$ & M1: First coating at Site. End & 7 \\
\hline $2021-07-22$ & M1: First coating at Site. Start & 1 \\
\hline $2021-07-21$ & AIV: Integration at Site. M1 assembly. End & 150 \\
\hline $2021-02-21$ & AIV: Integration at Site. M1 assembly. Start & 1 \\
\hline $2021-02-20$ & AIV: Telescope mechanics. Integration at Site. End & 60 \\
\hline $2020-12-22$ & AIV: Telescope. PAR & 240 \\
\hline $2020-04-26$ & AIV: Telescope mechanics. Integration at Site. Start & 42 \\
\hline $2020-03-15$ & MAIT: Telescope. End & 60 \\
\hline $2020-03-15$ & MAIT: Telescope. PSR & 540 \\
\hline $2020-03-15$ & MAIT: Telescope. Start & 1 \\
\hline $2018-07-23$ & DD: Telescope. End & 90 \\
\hline $2018-04-24$ & DD: Telescope. CDR & 300 \\
\hline $2017-06-28$ & DD: Telescope. Start & 1 \\
\hline $2017-06-27$ & PD: Telescope. PDR. End of process. & 90 \\
\hline $2017-03-29$ & PD: Telescope. PDR. End & 28 \\
\hline $2017-03-27$ & PD: Telescope. PDR. Start & 30 \\
\hline $2017-02-27$ & PD: Telescope. Final Documentation Package. Delivery to PDR panel. & 180 \\
\hline $2017-01-28$ & PD: Telescope. Final Documentation Package. Internal delivery. & 1 \\
\hline $2016-08-01$ & PD: Telescope. Start & 180 \\
\hline $2016-07-31$ & EPR: Telescope. Final Documentation Delivery $(3 / 3)$ & 320 \\
\hline $2016-02-02$ & EPR: Telescope. To & 3 \\
\hline & Total path (days) & 1 \\
\hline
\end{tabular}

[5] Echevarría, J., Tapia, M., Costero, R., Salas, L., Michel, R., and et al., "Site Testing at Observatorio Astronómico Nacional in San Pedro Mártir," Revista Mexicana de Astronomía y Astrofísica 37, 187-200 (2001).

[6] Schuster, W. J. and Parrao, L., "The Atmospheric Extinction of San Pedro Mártir," Revista Mexicana de Astronomía y Astrofísica 116, 682-692 (2001).

[7] Michel, R., Hiriart, D., and Chapela, A., "Four years of meteorological measurements at San Pedro Mártir 
Observatory," in [San Pedro Mártir: Astronomical Site Evaluation], Cruz-González, I., Avila, R., and Tapia, M., eds., Revista Mexicana de Astronomía y Astrofísica (Serie de Conferencias) 19, 99-102 (2003).

[8] Avila, R., Masciadri, E., Vernin, J., and Sánchez, L. J., "Generalized SCIDAR measurements at San Pedro Mártir. I. Turbulence profile statistics," Publications of the Astronomical Society of the Pacific 116, 682-692 (2004).

[9] Tapia, M., Hiriart, D., Richer, M., and Cruz-González, I., "Site testing at San Pedro Mártir," in [Workshop on Astronomical Site Evaluation], Cruz-González, I., Echevarria, J., and Hiriart, D., eds., Revista Mexicana de Astronomía y Astrofísica (Serie de Conferencias) 31, 47-60 (2007).

[10] Bohigas, J. and nez, J. M. N., "The green potential of the San Pedro Mártir Observatory," Revista Mexicana de Astronomía y Astrofísica 46, 89-107 (2010).

[11] Carrasco, E., nana, A. C., Sánchez, L. J., Avila, R., and Cruz-González, I., "An estimate of the temporal fraction of cloud cover at San Pedro Mártir Observatory," Monthly Notices of the Royal Astronomical Society 420, 1273-1280 (2012).

[12] Schock, M., Els, S., Riddle, R., Skidmore, W., Travouillon, T., and et al., "Thirty Meter Telescope site testing I: Overview," Publications of the Astronomical Society of the Pacific 121, 1151-1166 (2009).

[13] Skidmore, W., Els, S., Travouillon, T., Riddle, R., Schock, M., and et al., "Thirty Meter Telescope site testing V: Seeing and isoplanatic angle," Publications of the Astronomical Society of the Pacific 121, 11511166 (2009).

[14] Els, S. G., Travouillon, T., Schock, M., Riddle, R., Skidmore, W., and et al., "Thirty Meter Telescope site testing VI: Turbulence profiles," Publications of the Astronomical Society of the Pacific 121, 527-543 (2009).

[15] Otárola, A., Travouillon, T., Schock, M., Els, S., Riddle, R., and et al., "Thirty Meter Telescope site testing X: Precipitable water vapor," Publications of the Astronomical Society of the Pacific 122, 470-484 (2010).

[16] Yoshii, Y., Doi, M., Kohno, K., Miyata, T., Motohara, K., and et al., "Overview of University of Tokyo Atacama Observatory 6.5m telescope project," in [Ground-based and Airborne Telescopes V], Stepp, L. M., Gilmozzi, R., and Hall, H. J., eds., Proc. of SPIE 9145, 914507 (2014).

[17] Szentgyorgyi, A., McLeod, B., Fabricant, D., Fata, R., Norton, T., and et al., "The f/5 instrumentation suite for the Clay Telescope," in [Ground-based and Airborne Instrumentation for Astronomy IV], McLean, I. S., Ramsay, S. K., and Takami, H., eds., Proc. of SPIE 8446, 844628 (2012).

[18] Gunnels, S. M. and Carr, D. M., "Design of the Magellan Project 6.5 meter telescope: Telescope structure and mechanical systems," in [Advanced Technology Optical Telescopes V], Stepp, L. M., ed., Proc. of SPIE 2199, 414-427 (1994).

[19] Toledo-Ramírez, G., Reyes-Elias, N., Uribe, J. A., Martínez, C., Caballero, X., and et al., "San Pedro Mártir Telescope: Mexican design endeavour," in [Ground-based and Airborne Telescopes VI], Hall, H. J., Gilmozzi, R., and Marshall, H. K., eds., Proc. of SPIE 9906 (2016).

[20] Teran, J., Lee, W. H., Richer, M. G., Sánchez, B. S., Urdaibay, D., and et al., "Telescopio San Pedro Mártir Observatory preliminary design and project approach," in [Ground-based and Airborne Telescopes VI], Hall, H. J., Gilmozzi, R., and Marshall, H. K., eds., Proc. of SPIE 9906 (2016).

[21] Filgueira, J. M., Bec, M., Soto, J., Liu, N., and Peng, C. Y., "GMT software and controls overview," in [Software and Cyberinfrastructure for Astronomy II], Radziwill, N. M. and Chiozzi, G., eds., Proc. of SPIE 8451, 845111 (2012).

[22] Schectman, S. A., "The Magellan Project," in [Telescope Structures, Enclosures, Controls, Assembly/Integration/Validation, and Commissioning], Sebring, T. A. and Andersen, T., eds., Proc. of SPIE 4004, 47-56 (2000).

[23] Mcleod, B., Geary, J., Conroy, M., Fabricant, D., Ordway, M., and et al., "Megacam: A wide-field CCD imager for the MMT and Magellan," Publications of the Astronomical Society of the Pacific 127, 366-382 (2015).

[24] Mcleod, B., Fabricant, D., Nystrom, G., McCracken, K., Amato, S., and et al., "MMT and Magellan Infrared Spectrograph," Publications of the Astronomical Society of the Pacific 124, 1318-1335 (2012).

[25] Filgueira, J. M., Bec, M., Liu, N., Peng, C., and Soto, J., "End-to-end observatory software modeling using domain specific languages," in [Software and Cyberinfrastructure for Astronomy III], Chiozzi, G. and Radziwill, N. M., eds., Proc. of SPIE 9152, 915210 (2014). 